\title{
Propuesta del diseño de un sistema automatizado de bajo costo aplicado en la técnica Z-Scan
}

\section{Proposal for the design of a low-cost automated system applied in the Z-Scan technique}

\author{
R. Hernández ${ }^{1}$ | R. Ponce ${ }^{2}$ | J. Fiallos $^{3}$ | R. Mejía ${ }^{4}$
}

Recibido: 10 de junio de 2019/ Aceptado: 26 de noviembre de 2019

En el presente trabajo se presenta una propuesta de diseño para la automatización e implementación de la técnica Z-Scan. Pretendiendo se considere como base y con opción a modificación según las circustancias particulares bajo las cuales se implemente.

In the present work a design proposal for the automation and the implementation of the Z-Scan computer is presented. Pretending is considered as a basis and with the option of modification according to the particular circumstances in which it is implemented.

\section{PALABRAS CLAVES}

Técnica Z-Scan, transmitancia, óptica no lineal, coeficiente de absorción no lineal, 'indice de refracción, suceptibilidad eléctrica, microcontrolador, sistema de adquisición de datos (S.A.D.), configuración abierta configuración cerrada.

\section{KEYWORDS}

Z-Scan Technique, Transmitance,Nonlinear Optics, Nonlinear absortion coefficient, refraction index, electrical susceptibility, Microcontroller, Data acquisition system, closed configuration, opened configuration.

\footnotetext{
${ }^{1}$ Escuela de Física, Facultad de Ciencias, Universidad Nacional Autónoma de Honduras email: rene.hernandez@unah.edu.hn

${ }^{2}$ Escuela de Física, Facultad de Ciencias, Universidad Nacional Autónoma de Honduras email:rponce@unah.edu.hn

${ }^{3}$ Escuela de Física, Facultad de Ciencias, Universidad Nacional Autónoma de Honduras email: jonathan.fiallos@unah.edu.hn

${ }^{4}$ Escuela de Física, Facultad de Ciencias, Universidad Nacional Autónoma de Honduras email:roberto.mejia@unah.edu.hn
}

PACS

42.60.-V 


\section{I | INTRODUCCIÓN}

$\mathrm{L}$

A técnica Z-Scan es un método económico y de fácil implementación para el análisis de los parámetros ópticos no lineales de un material, comparado con la dificultad y costo que presentan los demás métodos para dicho análisis; por tal motivo la construcción y automatización de la técnica Z-Scan es la alternativa más utilizada. En este trabajo se presenta una propuesta utilizando componentes electrónicos de bajo costo para automatizar el montaje experimental. La implementación presentada consiste en construir un sistema físico donde se puede aplicar la técnica Z-Scan y un sistema programado (software) que permita controlar y automatizar los mecanismos que utiliza el sistema.

\section{II | TÉCNICA Z-SCAN}

La técnica Z-Scan se basa en el efecto de autoenfocamiento o autodesenfoque, causado por las propiedades ópticas no lineales de ciertos materiales y consiste en el análisis de la distorsión espacial que sufre el haz de un láser, que se hace pasar a través de una muestra (Racedo, 2015). Con la técnica ZScan se puede obtener de forma indirecta el índice de refracción y el coeficiente de absorción no lineal, así como también la suceptibilidad eléctrica no lineal de tercer orden. Esta técnica fue desarrollada por Sheik-Bahae et al. (Sheik-Bahae, Said, Wei, Hagan, y Van Stryland, 1990) en 1989. La ventaja que presenta la técnica Z-Scan es relacionar de forma simple la trasmitancia con la diferencia de fase, en la dirección de propagación del haz del láser, producto de los efectos no lineales; además de ser una técnica con muchas ventajas en cuanto a la precisión de sus mediciones (Sheik-Bahae y cols., 1990, Yan, Liu, Zhang, Zhou, y Tian, 2009).

\section{1) Óptica No Lineal (NLO)}

La NLO (por sus siglas en ingles) se ha interesado, en las últimas décadas, en la investigación y caracterizacón de diversos materiales con comportamiento no lineal. Los materiales con propiedades ópticas no lineales son utilizados en diversas aplicaciones tales como: comunicación óptica, optoelectrónica, instrumentación óptica, etc.

La NLO estudia fenómenos en los cuales, la interacción de la luz con la materia, hace cambiar sus propiedades ópticas. Los efectos ópticos no lineales se presentan cuando la magnitud del campo eléctrico que compone la luz es lo suficientemente intensa, comparable con la intensidad del campo eléctrico interno generado por un electrón.

El origen físico de los fenómenos ópticos no lineales surge de generalizar el vector polarización que es inducido por un campo eléctrico externo cuando interacciona con la materia. En la óptica lineal la polarización se escribe como:

$$
\tilde{P}(t)=\varepsilon_{0} \chi^{(1)} \tilde{E}(t)
$$

esta ecuación describe los fenómenos relacionados con la interacción de la luz con materiales que no modifican sus propiedades ópticas, mientras que en la óptica no lineal la polarización se expresa como una serie de potencias del campo eléctrico:

$$
P(t)=\varepsilon_{0}\left[\chi^{(1)} \tilde{E}(t)+\chi^{(2)} \tilde{E}^{2}(t)+\chi^{(3)} \tilde{E}^{3}(t)+\ldots\right]
$$

Donde $\chi^{(2)}$ y $\chi^{(3)}$ son conocidos como la susceptibilidad eléctrica de segundo y tercer orden, respecti- 


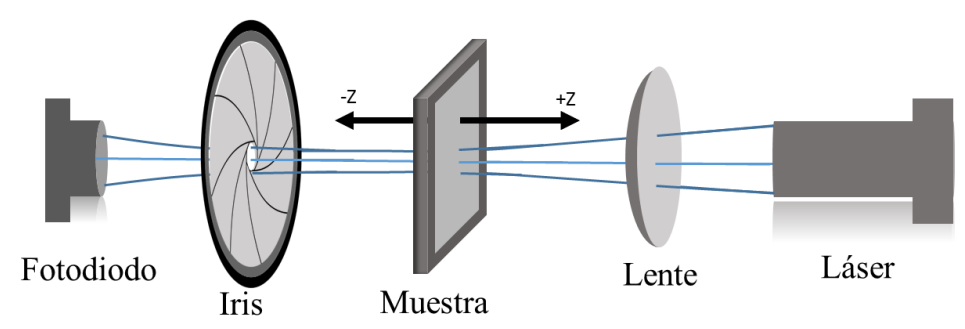

Figura 1: Diagrama que muestra la configuración del montaje experimental de la técnica Z-Scan

vamente.

Al considerar la ecuación anterior, surgen nuevos parámetros ópticos tales como: el índice de refracción no lineal $\left(n_{2}\right)$, el coeficiente de absorción no lineal $(\beta)$ y la susceptibilidad óptica $\chi^{(3)}$.

\section{PRINCIPIO DE FUNCIONAMIENTO DE LA TÉCNICA Z-SCAN}

Como se observa en la figura 1, la técnica Z-Scan consiste en correr una muestra con propiedades ópticas no lineales (principalmente los materiales de origen orgánico) a lo largo de la cintura de un haz con distribución Gaussiana y midiendo la intensidad transmitida a través de la muestra. Se espera que al desplazar la muestra a lo largo del eje de propagación del haz exista una variación en la intensidad del haz transmitido debido a efectos ópticos no lineales.

La técnica Z-Scan utiliza la cantidad física llamada transmitancia normalizada, que se define de forma general como la fracción de luz incidente que logra pasar a través de una muestra (Chiluiza, Piguave, y Yapur. 2011), para eliminar los efectos lineales y solamente observar los efectos no lineales (Racedo, 2015). La expresión matemática para la transmitancia es:

$$
T=\frac{I}{I_{0}}
$$

Donde: $I$ es la intensidad de la luz que sale del material e $I_{0}$ la intensidad de la luz que incide en el material.

Existen dos tipos de configuración para la realización de la técnica Z-Scan, la configuración abierta y la configuración cerrada. En la configuración cerrada se hace uso de un iris (ver fig[1), en la configuración abierta el iris es removido. 


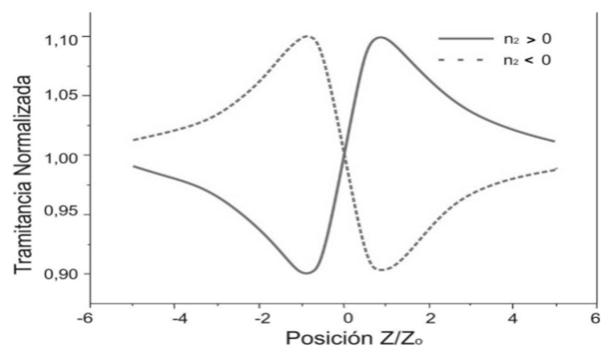

Figura 2: Curvas carácteristicas de la transmitancia para los casos donde el índice de refración no lineal es positivo y negativo (imagen tomada de Racedo (2015)).

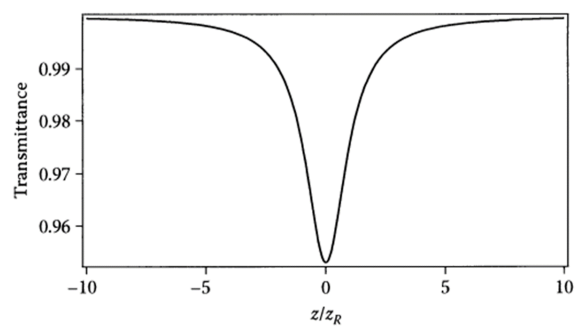

Figura 3: Gráfica donde se observa la curva característica para la transmitancia normalizada, cuando se utiliza la configuración abierta (imagen tomada de Power (2011)).

\section{1। Configuración cerrada}

Con esta configuración se obtiene el índice de refracción no lineal, el cual puede ser positivo o negativo. Dependiendo del signo del índice de refracción se obtienen curvas características diferentes para la transmitancia normalizada en función de la posición de la muestra dentro del montaje. En esta configuración la función del iris es hacer que la curva de transmitancia refleje sensibilidad a la distorsión del haz debido a las variaciones de intensidad del mismo.

En la Figura 2, la curva continua representa la forma característica cuando el índice de refracción no lineal es positivo, la curva punteada representa la forma característica cuando el índice de refracción no lineal es negativo. El origen de los picos en ambas curvas se debe a la variación de la intensidad, causado por la distorsión espacial que sufre el haz de luz que atraviesa la muestra a medida se desplaza en la dirección de propagación del haz; observando que la distorsión ocurre en distancias cercanas al punto focal.

\section{2 | Configuración abierta}

Con esta configuración se obtiene el coeficiente de absorción no lineal. Cuando el iris es removido la curva de transmitancia es sensible a la absorción no lineal e insensible a la distorsión del haz, cuyo efecto se observa en la forma de la curva característica de la transmitancia, ya que el pico es suprimido y el valle es acentuado, como se observa en la Figura 3.

\section{I PROPUESTA DEL DISEÑO DEL SISTEMA AUTOMATIZADO DE BAJO COSTO}

Como se puede observar en la Figura 1 para la automatización de la técnica se necesita controlar la posición de la muestra a lo largo del eje de propopagación del haz láser y en cada posición recolectar datos de la intensidad del haz transmitida por la muestra. Para lograr lo mencionado anteriormente se puede considerar diseñar un sistema automatizado. 


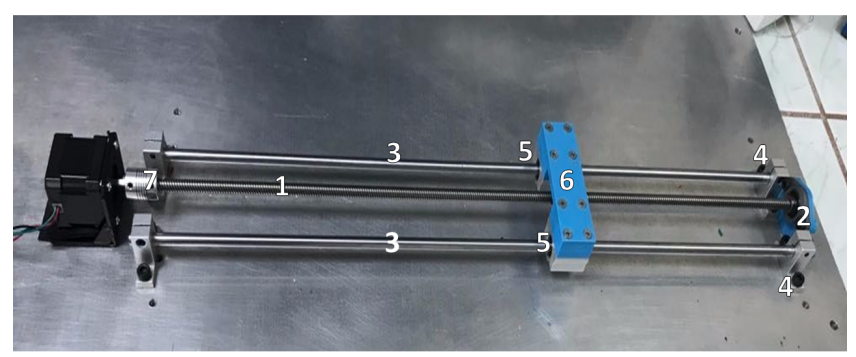

Figura 4: Propuesta de diseño para el riel de desplazamiento.

\section{1| Hardware}

El diseño de la parte física da la capacidad de control de la posición de la muestra y recolección de las cantidades físicas necesarias, esto será posible utilizando diversos dispositivos electrónicos. Esta sección del sistema a su vez se puede dividir en 3 partes: el riel, el motor de pasos y un sistema de adquisición de datos (SAD).

\section{1 | Riel}

Permite el movimiento de la muestra, acotando dicho movimiento a lo largo de la propagación del haz. La Figura 4 muestra el riel de desplazamiento. El riel consiste en:

1) Un tornillo de avance (1) unido a una tuerca de latón, donde uno de sus extremos descansa sobre un soporte de rodamiento (2). Estos componentes permiten controlar el movimiento de la muestra, y por ende la posición. Con un motor de pasos la muestra (6) puede ser trasladada en ambos sentidos: izquierda o derecha.

2) Una plataforma (6) que se encuentra adherida en sus extremos a dos bloques deslizantes (5), dichos bloques se hacen pasar a través de dos varillas lisas de acero (3) que están sostenidos en sus extremos por soportes (4). La plataforma permite colocar de forma adecuada la muestra que se quiere analizar con la técnica Z-Scan, además ésta se encuentra unida a la tuerca de latón. Las varillas de acero permiten mantener la dirección de movimiento de la plataforma.

\section{2 | Motor paso a paso}

La disposición del motor de paso se puede observar en la Figura 4. Al eje del motor se sujeta un acoplador flexible (7) que está unido al tornillo de avance, de esta forma al hacer girar el eje del motor se permite un deslizamiento suave y con baja tensión en el tornillo y puntos fijos. Para el adecuado funcionamiento del motor resulta necesario implementar un circuito que ayude a controlarlo, por tal razón se puede considerar como componentes del ciruito un microcontrolador y un driver para motores paso a paso. 


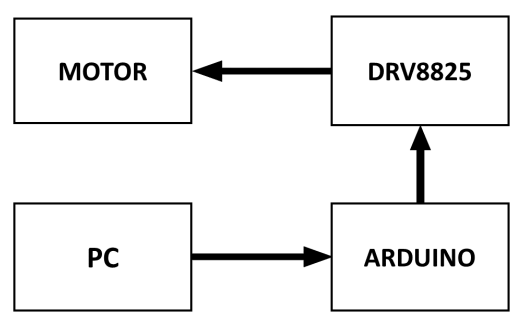

Figura 5: Diagrama general del circuito para el control de un motor paso a paso.

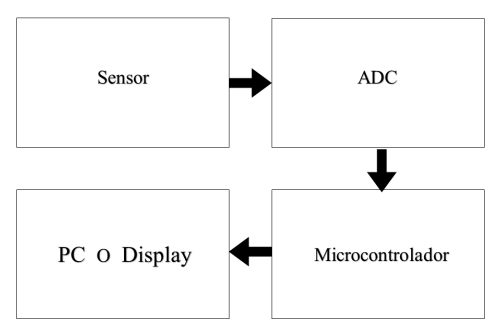

Figura 7: Diagrama de bloques del SAD (Jonathan Fiallos, 2017).

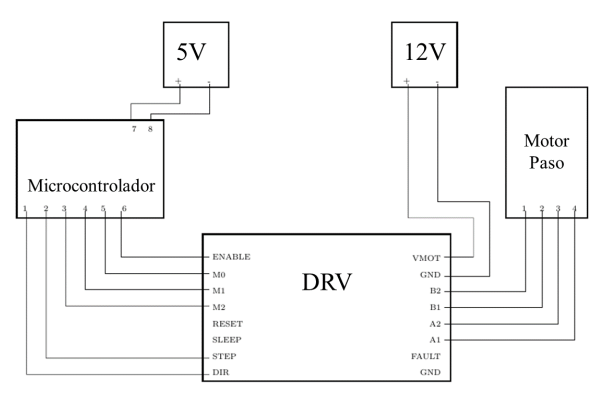

Figura 6: Conexión para el uso del driver DRV8825.

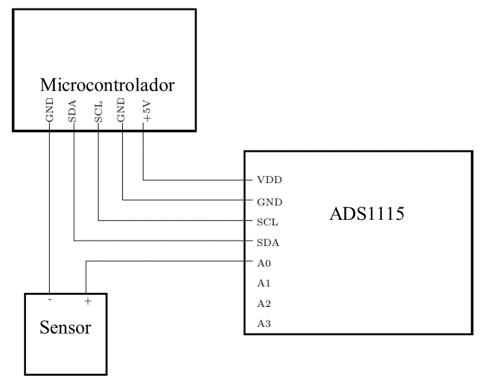

Figura 8: Circuito de conexión para el uso del convertidor analógico digital ADS1115

El diagrama de la Figura 5 muestra la forma en que el motor es manejado por un microcontrolador (arduino) y el driver DRV8825.

Con el driver se puede cambiar facilmente la dirección del giro del eje y la resolución del paso (hacer más pequeño el ángulo de giro del eje), pero es el microcontrolador el que permite al experimentador configurar el driver para manipular de forma conveniente el motor. El diagrama de la Figura 5 muestra que a traves de un PC el usuario puede comunicarse con el microcontrolador para cofigurar el driver y así controlar el motor de paso. 1

En el caso particular, se escogió como driver el DRV8825 "DRV8825StepperMotorControllerIC")(s.f.) cuyas características permiten tener una resolución máxima de $(1 / 32)^{\circ}$ del paso del motor; para un motor de 200 pasos o $1.8^{\circ}$ por pasos, con el driver se consigue una resolución máxima de $0.05625^{\circ}$ por paso del motor. La Figura 6 muestra las conexiones necesarias para el uso del driver junto al arduino.

\subsection{Sistemas de adquisición de datos}

Para construir el SAD se puede utilizar el modelo descrito en el diagrama anterior, el cual muestra las partes que conformarían el SAD. El sensor necesario para este SAD debe tener la característica de

\footnotetext{
${ }^{1}$ Se considera necesario mostrar el diagrama de bloque del circuito (fig 5 ya que existen diversos microcontroladores y drivers que pueden ser utilizados, los detalles de programación y conexión son particulares tanto al microcontrolador y al driver elegido.
} 


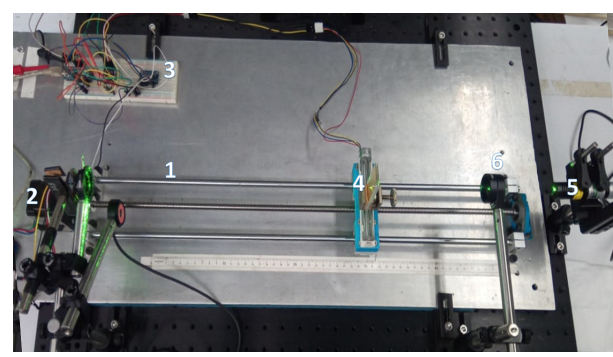

Figura 9: Imagen que muestra la integración y el funcionamiento del riel, el motor de pasos y el sistema de adquisición de datos.

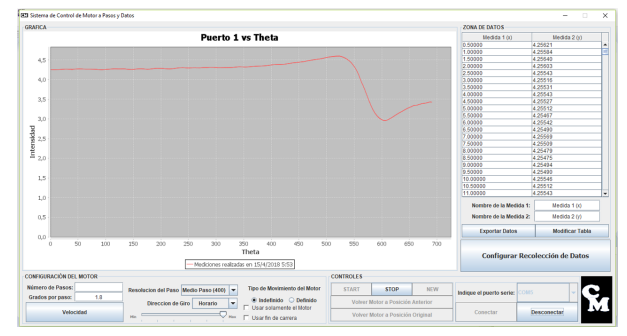

Figura 10: Interfaz gráfica de usuario programada.

registrar variaciones de la intensidad luminosa (Jonathan Fiallos 2017), para ello se utiliza el fotodiodo de área ancha FDS10X10 del fabricante Thorlabs ("Silicon Photodiode FDS10X10", s.f.).

El Conversor Análogo-Digital (CAD) es el encargado de convertir las señales análogas recibidas del sensor en señales digitales, las cuales son transmitidas a un microcontrolador y éste a su vez muestra los datos recibidos por el sensor en pantalla a través de un PC. 2 El CAD usado es el ADS1115 ("Ultra-Small,Low-Power,16-BitAnalog-to-DigitalConverterwithInternalReference", s.f.) que cuenta con una resolución de 16 bit.

Como se muestra en la Figura 9, para el hardware en términos generales se debe diseñar un riel (1) que mediante un motor paso a paso (2) permita el movimiento controlado de una muestra y un sistema de recolección de datos SAD (3). Además se debe diseñar un circuito que faculte el control del motor de paso y éste se encuentre acoplado al SAD para la recolección de datos, realizando ambas tareas de forma simultánea. Lo mencionado anteriormente garantiza el movimiento de la muestra (4) a lo largo de la cintura del haz láser (5) que se hace pasar a través de una lente (6), automatizando de esta manera la técnica Z-Scan.

\section{2| Software}

Ya diseñada la parte física del sistema es necesario crear un programa que permita el fácil manejo del sistema al experimentador. El diseño del programa tiene dos partes: primero la programación del microcontrolador que tiene la lógica para hacer funcionar de forma adecuada el motor, la recepción y envío de datos recolectados; segundo, programación de una interfaz gráfica donde el usuario podrá interactuar con el microcontrolador y recibir los datos de las diferentes mediciones de magnitudes físicas.

Para programar el microcontrolador (Arduino) se creó una librería propia. La librería contiene toda la lógica para: mover y parar el motor, mover el motor por una cantidad de pasos determinada, cambiar la dirección de giro, cambiar la resolución del paso, y tomar datos por medio de sensores ${ }^{3}$

\footnotetext{
2 Para más detalles revisar el modelo completo en Jonathan Fiallos. 2017,

${ }^{3}$ Para que el microcontrolador realice todas las acciones descritas, es necesario establecer una comunicación con él, mediante el puerto serial de la computadora; donde la interfaz gráfica de usuario pueda: enviar la acción que se desea ejecutar y recibir los datos recolectados por el ADC.
} 


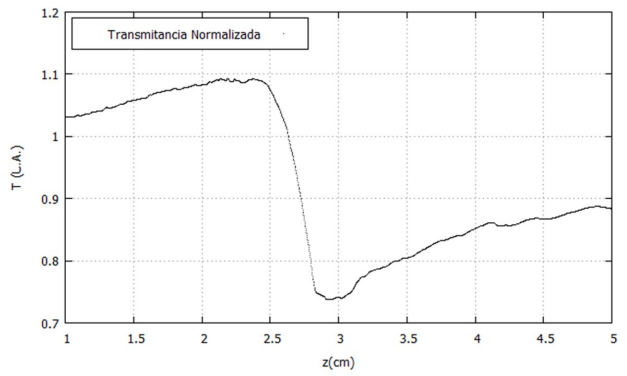

Figura 11: Imagen que muestra los resultados experimentales obtenidos utilizando el aceite de ajonjoli en configuración cerrada.

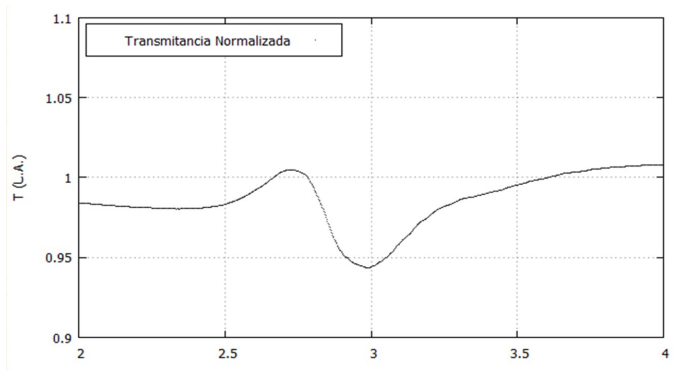

Figura 12: Imagen que muestra los resultados experimentales obtenidos utilizando el aceite de ajonjolí en configuración abierta.

Por último, se programa una interfaz gráfica para mostrar todas las opciones posibles al experimentador, creando un software ajustado a las especificaciones requeridas para la aplicación adecuada de la técnica Z-Scan. El programa, realizado, permite al usuario: controlar el motor de pasos con o sin el acompañamiento de la recolección de datos, ajustar la resolución y dirección de giro del motor, realizar cálculos sencillos con los datos obtenidos, exportar los datos y visualizarlos en tiempo real. Además, se añadió opciones adicionales al software, como la posibilidad para el usuario de configurar el ADC-1115, para conseguir utilizar todas las características que ofrece.

\section{I RESULTADOS}

La curva de transmitancia es clave en la tecniza Z-Scan debido que a partir de ella es posible determinar los parámetros ópticos no lineales de distintas sustancias. Las curvas que se esperan obtener del diseño propuesto tendrán características similares con las figuras 2 y 3 Las gráficas mostradas a continuación representan las curvas de transmitancia obtenidas para diferentes barridos utilizando aceite de oliva y de ajonjolí, una lente biconvexa con foco de $3 \mathrm{~cm}$ y un láser de aproximadamente $100 \mathrm{~mW}$ de potencia.

Al analizar cualitativamente las curvas obtenidas, se aprecia un comportamiento similar al de las gráficas en las figuras 2 y 3 . Sin embargo, en las figuras 12 y 14 pertenecientes a la configuración abierta del Z-Scan, se puede apreciar un pico antes del valle, debido a efectos térmicos que hacen que la curva no presente simetría (Marbello, Valbuena, y Racedo, 2019).

Para verificar que las curvas obtenidas tienen el comportamiento adecuado, para el aceite de oliva y ajonjolí, estas pueden ser comparadas con las curvas de transmitancia obtenidas en otros trabajos, que muestran para ambos aceites el coeficiente de refracción no lineal es negativo; ya que en las curvas de transmitancia para la configuración cerrada, se presenta primero un pico seguido de un valle. Este mismo comportamiento se presentan en las curvas de transmitancia obtenidas con el montaje automatizado propuesto (comparar las figuras 11 y 13 con las figuras con 15 y 17]).

A continuación se muestran las curvas de transmitancias obtenidas, para el aceite de oliva y ajonjolí (sésamo), en el trabajo: "Non-linear optical response of edible oils by means of the Z-scan technique" (Marbello y cols. 2019). 


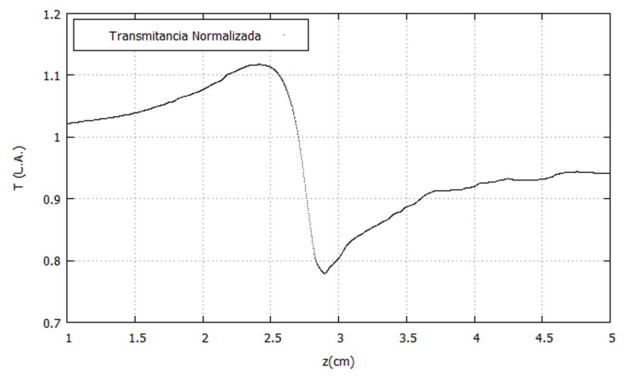

Figura 13: Imagen que muestra los resultados experimentales obtenidos utilizando el aceite de oliva configuración cerrada.

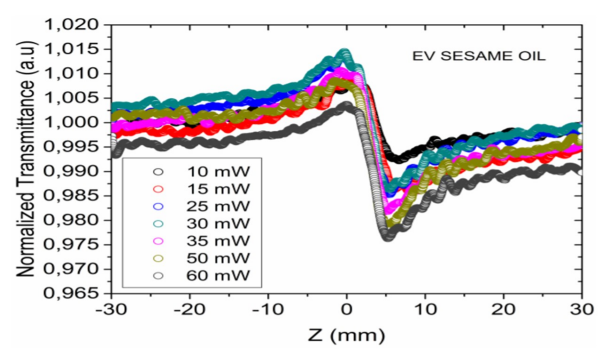

Figura 15: Imagen que muestra los resultados experimentales previos obtenidos utilizando el aceite de ajonjolí en configuración cerrada, a diferentes valores de potencia del haz láser (imagen tomada de Marbello y cols. (2019))

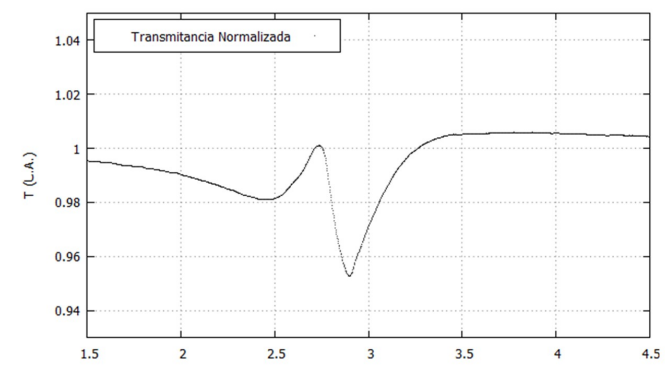

Figura 14: Imagen que muestra los resultados experimentales obtenidos utilizando el aceite de oliva configuración abierta.

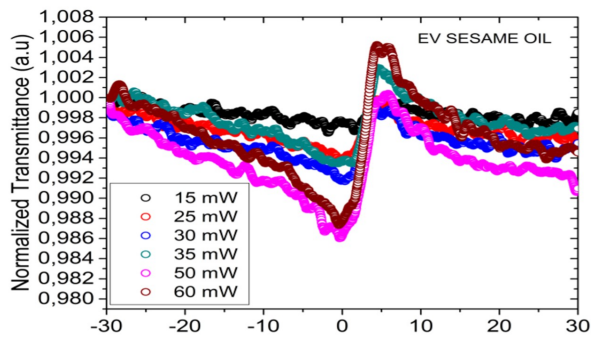

Figura 16: Imagen que muestra los resultados experimentales previos obtenidos utilizando el aceite de ajonjolí en configuración abierta, a diferentes valores de potencia del haz láser (imagen tomada de Marbello y cols. (2019)).

Se puede observar que las curvas de transmitancia obtenidas con el montaje propuesto para la configuración abierta son similares a las reportadas en (Marbello y cols. 2019), excepto para el aceite de ajonjolí (comparar las figuras 12 y 16). La diferencia consiste en que la forma de la curva obtenida aparece invertida respecto a la reportada en (Marbello y cols., 2019); esta puede ser explicada en que la muestra fue desplazada en sentido contrario al desplazamiento realizado para obtener las curvas mostradas en las figuras 11, 13, 14, por ende se obtiene una curva con la misma forma pero invertida respecto a la curva reportada en (Marbello y cols. 2019).

\section{VI | CONCLUSIONES}

Se puede concluir que la propuesta de diseño presentada es una alternativa viable para la implementación de la técnica Z-Scan, ya que permite obtener las curvas de transmitancia, concordando con el comportamiento esperado al examinar de forma cualitativa los resultados experimentales con las curvas de transmitancia reportadas por otros investigadores. La inversión aproximada para la realización del montaje experimental que abarca: el riel, el motor de pasos y los componentes electrónicos del S.A.D. fue alrededor de USD 325. 


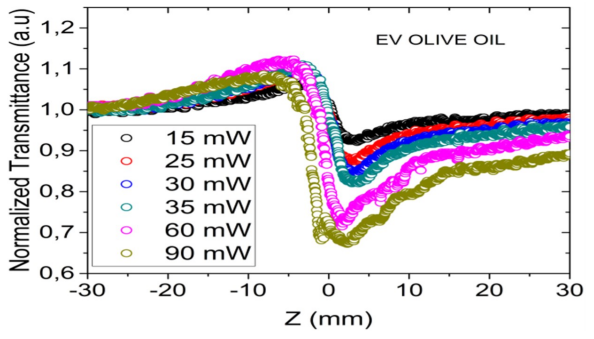

Figura 17: Imagen que muestra los resultados experimentales previos obtenidos utilizando el aceite de oliva configuración cerrada, a diferentes valores de potencia del haz láser (imagen tomada de Marbello y cols. (2019)).

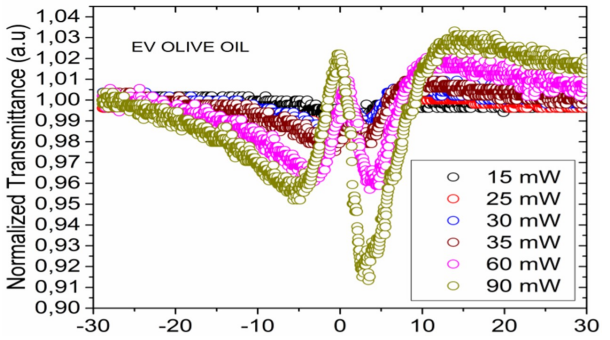

Figura 18: Imagen que muestra los resultados experimentales previos obtenidos utilizando el aceite de oliva configuración abierta, a diferentes valores de potencia del haz láser (imagen tomada de Marbello y cols. (2019)).

\section{REFERENCIAS}

Chiluiza, K., Piguave, F., y Yapur, I. M. (2011). Análisis, diseño y construcción de un colorímetro básico, para ser utilizado en un laboratorio clínico, utilizando microcontroladores.

Drv8825steppermotorcontrolleric [Manual de software informático]. (s.f.).

Jonathan Fiallos, R. P. y. R. M., René David Hernández. (2017). Sistemas de adquisición de datos aplicados en la medición de variables físicas. Revista de la Escuela de Física UNAH, V(2), 47-52.

Marbello, O., Valbuena, S., y Racedo, F. (2019). Non-linear optical response of edible oils by means of the z-scan technique. En Journal of physics: Conference series (Vol. 1219, p. 012008).

Power, P. E. (2011). Fundamental of nonlinear optics. CRC Press. (figura 8.15)

Racedo, A. G. S. V. R. S. F. (2015). Measurement of the nonlinear optical properties of olive oil using z-scan. Optica Pura y Aplicada, 48(1), 55-61.

Sheik-Bahae, M., Said, A. A., Wei, T.-H., Hagan, D. J., y Van Stryland, E. W. (1990). Sensitive measurement of optical nonlinearities using a single beam. IEEE journal of quantum electronics, 26(4), 760-769.

Silicon photodiode fds10x10 [Manual de software informático]. (s.f.).

Ultra-small,low-power,16-bitanalog-to-digitalconverterwithinternalreference [Manual de software informático]. (s.f.).

Yan, X.-Q., Liu, Z.-B., Zhang, X.-L., Zhou, W.-Y., y Tian, J.-G. (2009). Polarization dependence of z-scan measurement: theory and experiment. Optics express, 17(8), 6397-6406. 